\title{
Plant Regeneration of 'Valencia' Sweet Orange, 'Femminello' Lemon, and the Interspecific Somatic Hybrid following Protoplasm Fusion
}

\author{
N. Tusa ${ }^{1}$, J.W. Grosser ${ }^{2}$, and F.G. Gmitter, Jr. ${ }^{3}$ \\ Citrus Research and Education Center, University of Florida, Institute of Food and Agricultural \\ Sciences, Department of Fruit Crops, 700 Experiment Station Road, Lake Alfred, FL 33850 \\ Additional index words. $\quad$ somatic hybridization, citrus, polyploidy, plant breeding, Citrus sinensis, Citrus limon
}

\begin{abstract}
Protoplasm culture following the chemical fusion of 'Valencia' sweet orange [Citrus sinensis (L.) Osb.] protoplasts, isolated from an embryogenic suspension culture, with 'Femminello' lemon [Citrus limon (L.) Burro. f.] leaf protoplasts resulted in the regeneration of an interspecific allotetraploid somatic hybrid plant, two autotetraploid lemon plants, and diploid plants from both parents. The regeneration of plants from lemon leaf protoplasts is an example of protoplast-to-plant regeneration from non-nucellus-derived tissue for Citrus. Regenerated plants were classified according to leaf morphology, chromosome number, and analyses of phosphohexose isomerase (PHI), peroxidase (PER), and 6-phosphoglucose dehydrogenase (PGD) zymograms. The somatic hybrid plant was vigorous, with leaves morphologically intermediate to the parents. The tetraploid lemon plants were similar to diploids, although less vigorous and with thicker leaves. The tetraploid lemon and somatic hybrid plants, if fertile, could be used in interploid sexual crosses to breed triploid seedless lemon cultivars with tolerance of mal secco disease from sweet orange. Further investigation of plant regeneration from leaf protoplasts could increase the number of totipotent Citrus clones amenable to somatic hybridization and genetic transformation experiments.
\end{abstract}

Cultivar development in Citrus by conventional breeding methods has been limited by the complex reproductive biology of Citrus (Grosser and Gmitter, 1990b; Swingle and Reece, 1967). Recently, interspecific and intergeneric somatic citrus hybrids have been obtained via protoplasm fusion (Ohgawara et al., 1985, 1989; Grosser and Gmitter, 1990a; Grosser et al., 1988a, 1988b, 1989; Kobayashi and Ohgawara, 1988; Kobayashi et al., 1988). These successes suggest potential new gene transfer strategies for citrus scion and rootstock improvement (Grosser and Gmitter, 1990b).

Mal secco is a systemic fungal disease of Citrus caused by Phoma tracheiphila (Petri) Kantsch. \& Gik. and occurs widely in the Mediterranean and Black Sea areas (Solel and Salerno, 1988). Lemon and citron (C. medica L.) are most susceptible, but grapefruit (C. paradisi Macf.) and sweet orange are considered to be tolerant. The development of improved lemon cultivars with adequate tolerance or resistance to mal secco disease is an important breeding objective in these areas. In Italy, breeding efforts have produced lemon cultivars with improved tolerance to mal secco disease (including 'Monachello', 'Interdonato', and 'Santa Teresa'), but they lack the productivity and fruit quality of 'Femminello' (Salerno and Cutuli, 1977). Other high quality lemon selections are being evaluated but none apparently has adequate tolerance to this disease.

Our objective was to generate interspecific somatic hybrid plants of 'Femminello' lemon and 'Valencia' sweet orange in an effort to combine the good quality and performance of 'Femminello' lemon with the cold hardiness and mal secco-tolerance of 'Valencia'. Such an allotetraploid hybrid could be used as a breeding parent in tetraploid $\times$ diploid hybridization with lemon

Received for publication 30 Oct. 1989. Florida Agricultural Experiment Station Journal Series no. R-00216. The cost of publishing this paper was defrayed in part by the payment of page charges. Under postal regulations, this paper therefore must be hereby marked advertisement solely to indicate this fact.

'Researcher. Present address: Centro di Studio del C.N. R. per il Miglioramento Genetico degli Agrumi. Viale delle Scienze 90128 Palermo, Italy.

Associate Professor.

Assistant Professor. to produce improved seedless triploid lemon-types tolerant of mal secco.

\section{Materials and Methods}

Protoplasm isolation. Protoplasts of 'Femminello' lemon were isolated from leaves of young nucellar seedlings maintained in a growth chamber (16-hr photoperiod, $300 \mu \mathrm{mol} \cdot \mathrm{s}^{-1} \cdot \mathrm{m}^{-2}$ light intensity, 26 to $30 \mathrm{C}$ ), according to the procedure of Grosser and Chandler (1987). 'Valencia' protoplasts were isolated from a 1year-old suspension culture initiated from nucellus-derived embryogenic callus. The 'Valencia' suspension culture was maintained and subculture every 2 weeks on a modified MT basal medium (Murashige and Tucker, 1969) containing $500 \mathrm{mg}$ malt extract/liter and $50 \mathrm{~g}$ sucrose/liter. This medium, designated as $\mathrm{H}+\mathrm{H}$, contains a MT macronutrient regime modified by a $50 \%$ reduction in the $\mathrm{MT} \mathrm{NH}_{4} \mathrm{NO}_{3}$ and $\mathrm{KNO}_{3}$ concentrations and the addition of $1550 \mathrm{mg}$ glutamine/liter and $750 \mathrm{mg} \mathrm{KCl}$ / liter (Grosser and Gmitter, 1990b). For protoplasm isolation, 1 $\mathrm{ml}$ of suspension (4 to 12 days into the 14-day cycle) was transferred to a $60 \times 15-\mathrm{mm}$ petri dish with a widemouth pipet. The suspension medium was carefully removed with a pasteur pipet and replaced with $3 \mathrm{ml} \mathrm{BH} 3$ protoplasm culture medium (Grosser and Chandler, 1987; Grosser and Gmitter, 1990b). One milliliter of enzyme solution (Grosser and Chandler, 1987; Grosser and Gmitter, 1990b) was added drop by drop to facilitate cell separation, and the petri dishes were sealed with Nescofilm (Karlan Chemical, Torrance, Calif.) and incubated for $16 \mathrm{hr}$ in the dark at $28 \mathrm{C}$ on a rotary shaker $(50 \mathrm{rpm})$. Both protoplasm preparations were passed through a $45-\mu \mathrm{m}$ stainless steel filter and centrifuged for $10 \mathrm{~min}$ at $100 \mathrm{x} g$ to remove the enzymemedium solution. Protoplasts were purified further by centrifugation on a sucrose-mannitol gradient as described below. Pelleted protoplasts were gently resuspended in $4 \mathrm{ml}$ of a $25 \%$ sucrose solution containing CPW nutrients (Power et al., 1984). Two milliliters of 13'\% mannitol solution with CPW nutrients were carefully layered on top to avoid mixing. The preparations were then centrifuged $6 \mathrm{~min}$ at $100 \times \mathrm{g}$.

Protoplasm fusion. Approximately equal volumes of purified protoplasts were collected from the band at the interface of the 
two layers for each parent, mixed, and centrifuged 4 min at $100 \mathrm{x}$ g. Pelleted mixed protoplasts were resuspended in a total volume of $\mathrm{BH} 3$ medium equal to $10 \times$ the volume of the original pellet. Citrus suspension culture-derived protoplasts are generally larger than citrus leaf protoplasts, and the final mixture therefore contained a higher concentration of leaf protoplasts. Two drops of the resuspended protoplasm mixture were pipetted to the center of each of 12 fusion petri dishes $(60 \times 15 \mathrm{~mm})$, and the protoplasts were fused using the polyethylene glycol (PEG) method of Menczel et al. (1981) as modified by Grosser and Gmitter (1990b). Calculation of protoplasm densities before fusion is unnecessary with this method (Grosser and Gmitter, 1990b; Grosser et al., 1988a, 1988b, 1989).

Protoplasm culture and plant regeneration. After fusion, protoplasts were cultured directly in fusion petri dishes in 8 to 20 drops of a mixture of $1 \mathrm{BH} 3$ medium : 1 liquid MT basal medium (v/v) containing $200 \mathrm{~g}$ sucrose and $0.5 \mathrm{~g}$ malt extract/ liter. Fusion petri dishes were sealed with Nescofilm and stored in plastic containers in the dark at $28 \mathrm{C}$. After 5 weeks of incubation, cultures were supplemented with 10 to 12 drops of a mixture of $1 \mathrm{BH} 3$ medium : $1 \mathrm{MT}$ basal medium liquid (v/v) containing $125 \mathrm{~g}$ sucrose and $0.5 \mathrm{~g}$ malt extract/liter. After 2 more weeks, the osmoticum. was reduced further by the addition of $2 \mathrm{ml}$ of a mixture of $1 \mathrm{BH} 3$ medium : 1 liquid MT basal medium (v/v) containing $50 \mathrm{~g}$ sucrose and $0.5 \mathrm{~g}$ malt extract/ liter (designated EME, Grosser and Gmitter, 1990b). Developing colonies were transferred, by careful pouring, to $100 \mathrm{x}$ 15-mm petri dishes containing $0.8 \%$ agar (Sigma, St. Louis) solidified EME medium. After 3 to 5 weeks, developing somatic embryos were transferred to EME 1500 [EME plus an additional $1.0 \mathrm{~g}$ malt extract/liter; Grosser and Gmitter (1990b)] for enlargement. Large, well-shaped embryos were germinated on MT basal medium containing (per liter) $25 \mathrm{~g}$ sucrose, $1.0 \mathrm{mg}$ gibberellic acid $\left(\mathrm{GA}_{3}\right), 15 \mathrm{mg}$ coumarin, $0.02 \mathrm{mg}$ naphthaleneacetic acid (NAA), and $0.5 \mathrm{~g}$ malt extract (designated $\mathrm{B}+$ medium) (Grosser and Gmitter, 1990b). Embryos that developed shoots but poor or no roots were transferred to one-half strength MT basal medium containing (per liter) $25 \mathrm{~g}$ sucrose, $0.5 \mathrm{~g}$ neutralized activated. charcoal, and $0.02 \mathrm{mg}$ NAA (designated RMAN medium) (Grosser and Gmitter, 1990b) for taproot induction. Rooted plantlets were transferred to a commercial potting mixture containing $60 \%$ peat and $40 \%$ poly-beads and maintained at high humidity until acclimated.

Cytology. The chromosome number of root tip cells from regenerated plants was determined using the hematoxylin staining technique of Sass (1958), as modified by X. Ling (personal communication) (Grosser and Gmitter, 1990b). Active root tips were excised 3 to $4 \mathrm{hr}$ after the onset of light and placed for 2 $\mathrm{hr}$ in a supersaturated aqueous solution of 1,4-dichlorobenzene solution at $4 \mathrm{C}$. Then, root tips were rinsed in double-distilled (old) $\mathrm{H}_{2} \mathrm{O}$ and transferred to a solution of $3100 \%$ ethanol :1 acetic acid (v/v) for $2 \mathrm{hr}$ and hydrolyzed in $5 \mathrm{~N} \mathrm{HCl}$ for $20 \mathrm{~min}$. After another rinse in dd $\mathrm{H}_{2} \mathrm{O}$, root tips were transferred to a $4 \% \mathrm{Fe} \mathrm{NH} \mathrm{N}_{4}\left(\mathrm{SO}_{4}\right)_{2}$ solution, held there for $2 \mathrm{hr}$ and rinsed thoroughly by washing in dd $\mathrm{H}_{2} \mathrm{O}$ for at least 30 rein, and stained in a $0.2 \%$ hematoxylin solution for $30 \mathrm{~min}$. After a final rinse in $\mathrm{dd} \mathrm{H}_{2} \mathrm{O}$, root tips were squashed with a smooth round-tipped glass rod onto a glass slide in $45 \%$ acetic acid solution, spread under a cover slip, and examined. Preparations were scanned at $200 \times$ magnification, and chromosomes were counted at $1000 \mathrm{x}$ magnification under oil immersion. At least three root tips and four metaphase mitoses per root tip were examined for each regenerated plant.
Electrophoretic analysis of leaf isozymes. Seedling leaf tissue from 'Femminello', 'Valencia', and all plants obtained from protoplasm fusion and culture was sampled as in Torres et al. (1978). Isozymes were separated using horizontal starch gel electrophoresis on $0.8 \times 18.2 \times 18.2-\mathrm{cm}$ gels containing $10 \%$ Connaught starch (Fisher Scientific, Pittsburgh, Pa.) and O. 15\% agarose. Peroxidase (PER, EC 1.11.1.7), phosphohexose isomerase (PHI, EC 5.3.1.9), and 6-phosphogluconate dehydrogenase (PGD, EC 1.1.1.44) isozymes were resolved using the pH 5.7 histidine citrate buffer of Cardy et al. (1981). Electrophoresis was carried out for $3 \mathrm{hr}$ at $4 \mathrm{C}$ and constant $60-\mathrm{mA}$ current. Staining recipes were from Vallejos (1983).

\section{Results}

A total of 51 plants were regenerated from separate somatic embryos following protoplasm fusion. Variation in leaf shape and thickness (Fig. 1) and young leaf pigmentation was observed among the regenerated plants. Chromosome counts revealed that three of the regenerated plants were tetraploid $(2 \mathrm{n}=4 \mathrm{x}=36)$ (Fig. 2), but the remaining 48 were diploid $(2 \mathrm{n}=2 \mathrm{x}=18)$. One' of the tetraploid plants was vigorous and exhibited leaf morphology intermediate to that of the parents; it was presumed, therefore, to be an interspecific somatic hybrid (Fig. 1). The remaining two tetraploid plants were less vigorous and resembled 'Femminello' lemon; they exhibited thick, lemon-type leaves and red pigmentation in young shoots and leaves, a characteristic of lemon but not sweet orange. Morphologically, 20 of the
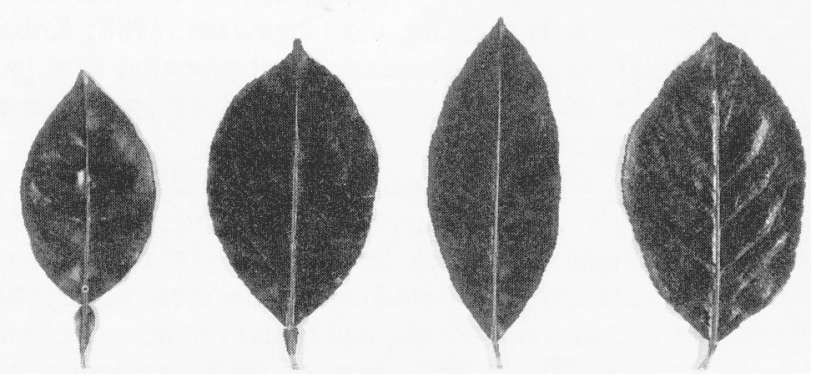

Fig. 1. Leaf morphology (from left to right) of 'Valencia' sweet orange, somatic hybrid of 'Valencia' + 'Femminello', diploid 'Femminello' lemon, and tetraploid 'Femminello' lemon. Leaves were taken from protoplast-derived plants $=18$ months old.



Fig. 2. Root tip squash prepared from the 'Valencia' + 'Femminello' somatic hybrid, showing a tetraploid cell $(2 n=4 x=36)$. Focal adjustments were necessary for an exact count (magnification = $800 \mathrm{x}$, oil immersion). 
diploid plants resembled 'Femminello' lemon and 28 resembled 'Valencia' sweet orange. These presumed identities were later verified by electrophoretic analysis of PHI, PER, and PGD isozymes. All regenerated plants survived transfer to soil and are still actively growing.

PHI provided the clearest evidence" of somatic hybridity. Genotypes at this locus were $M S$ for 'Valencia' (Torres et al., 1985) and WS for 'Femminello' (Torres et al., 1978). The banding pattern of the somatic hybrid was consistent with the expected MWSS genotype, displaying bands from both parents and a unique heterodimer $W M$ Staining activity of the $W W$ band was low but detectable (Figs. 3 and 4).

"'Valencia' was found to be $F F$, and 'Femminello' arid the somatic hybrid plant were $F S$ for PER (Fig. 5) (Moore and Castle, 1988). The staining intensity of the $F$ band in the somatic hybrid was greater than the $\mathrm{S}$ band because of the presence of three copies of the $F$ allele and only one copy of the $S$ allele per genome.

The alleles of PGD have not been defined, but it was possible to distinguish clearly sweet orange from lemon. 'Valencia' pro-

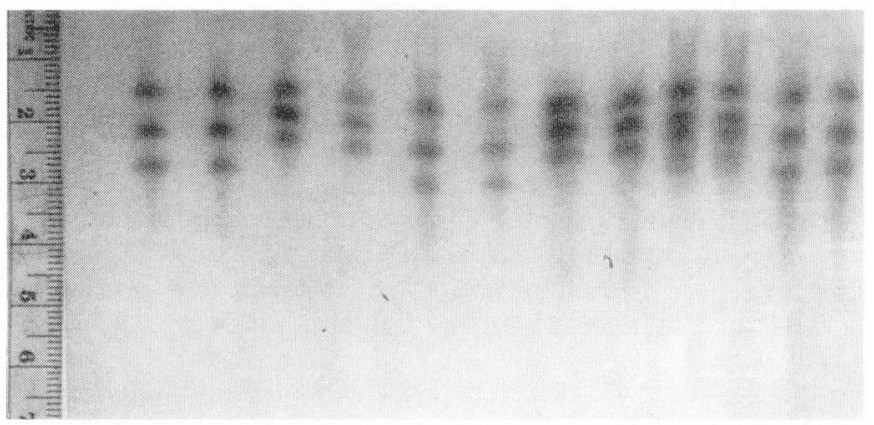

Fig. 3. Starch gel stained for PHI activity. From left to right, 'Femminello' from leaf protoplasts (lanes 1 and 2), 'Valencia' from protoplasts (lanes 3 and 4), tetraploid 'Femminello' lemon (lanes 5 and 6), 'Valencia' from a nucellar seedling (lanes 7 and 8), the somatic hybrid (lanes 9 and 10), and 'Femminello' from a nucellar seedling (lanes 11 and 12). Cathode is at top of figure.

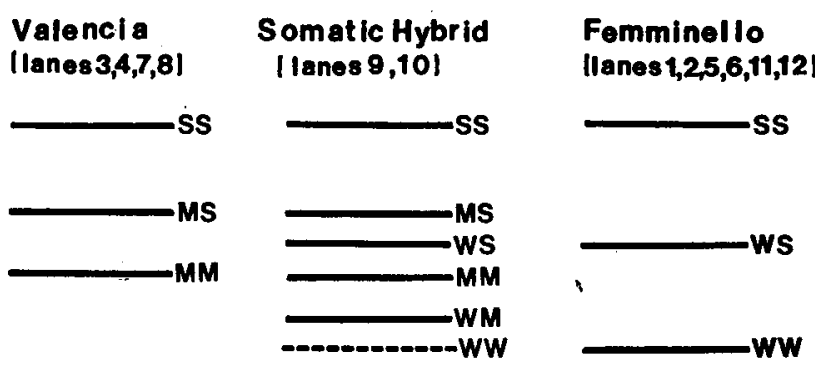

Fig. 4. Diagram of PHI activity, summarizing banding patterns and allelic identity of 'Valencia', the somatic hybrid, and 'Femminello' from the gel shown in Fig. 3 .

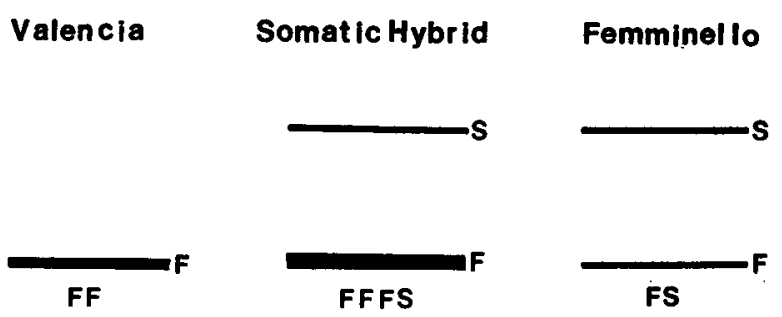

Fig. 5. Diagram of PER activity showing banding patterns and allelic identity of 'Valencia', the somatic hybrid, and 'Femminello'. duced five bands and 'Femminello' produced two bands that migrated the same distance as two of the 'Valencia' bands. The zymogram of the somatic hybrid resembled sweet orange, but staining intensity of the bands common to both parents was greater than the bands unique to sweet orange. The isozyme profiles of the two autotetraploid lemon plants were equivalent to that of 'Femminello' for all three enzyme systems.

\section{Discussion}

Our general strategy for citrus somatic hybridization has been to fuse protoplasts of one parent, isolated from habituated nucellus-derived tissue, with protoplasts of a second parent, isolated from nonembryogenic tissue that lacks the capacity for culture and regeneration in growth regulator-free protoplasm culture medium (usually leaf- or epicotyl-derived seedling callus). In our previous work, this approach eliminated whole plant recovery from the second parent. Hybrid cells resulting from fusion displayed an enhanced capacity for somatic embryogenesis and plant regeneration. Some of us reported previously that plant regeneration from unfused protoplasts of either parent, following fusions of embryogenic suspension culture-derived protoplasts with leaf protoplasts, was never observed (Grosser and Gmitter, 1990b; Grosser et al., 1988a, 1988b, 1989). In the present case, however, not only was an interspecific somatic hybrid produced, but also 28 diploid plants from unfused 'Valencia' sweet orange embryogenic callus-derived protoplasts, 20 diploid 'Femminello' lemon plants from unfused leaf protoplasts, and two tetraploid 'Femminello' lemon plants, also from leaf protoplasts. This surprising result could be the result of sucrose-mannitol gradient purification of protoplasts from both parents before fusion. We have not used this procedure in any of our previously reported experiments. This procedure reduces the amount of particulate debris and nonviable or damaged protoplasts, factors that could have a negative effect on protoplasm culture. These results also demonstrate that selection at the cellular level is not necessary for somatic hybrid plant recovery in citrus, even when plants are regenerated from unfused protoplasts of both parents.

This report of plant regeneration from Citrus leaf protoplasts is an additional one from non-nucellus-derived tissue. Recently, Ohgawara et al. (1989) reported the recovery of a few 'Marsh' grapefruit plants from unfused mesophyll-derived protoplasts following protoplasm fusion. We have also produced plants from unfused leaf protoplasts of other cultivars following protoplasm fusion, using the methods described here, including 'Duncan' grapefruit and 'Murcott' tangor (purported hybrid of C. sinensis and C. reticulate Blanco). These results show the potential for developing a leaf protoplasm to plant regeneration system as an alternative to embryogenic nucellus-derived callus or suspension cultures. Embryogenic callus lines of many important Citrus cultivars are often difficult or impossible to obtain, and totipotency in Citrus has been limited to a few polyembryonic clones (Grosser and Gmitter, 1990b), including 'Villafranca' lemon (Vardi et al., 1982). The development of an efficient protocol for plant regeneration from leaf protoplasts of monoembryonic and recalcitrant polyembryonic genotypes would greatly facilitate somatic hybridization and genetic transformation research. Preliminary observations indicate that co-culture is necessary for 'Femminello' leaf protoplasts to undergo mitosis in growth regulator-free protoplasm culture media. Both mitosis and somatic embryogenesis of 'Femminello' leaf protoplasm-derived cells probably are induced or stimulated by co-culturing with embryogenic 'Valencia' cells undergoing the same processes. 
Further experimentation is also needed to determine if the subjection of leaf protoplasts to PEG treatment is necessary or beneficial for subsequent plant regeneration.

Another unexpected result was the regeneration of two autotetraploid lemon plants. These plants may have resulted from homokaryotic fusions of 'Femminello' leaf protoplasts or from chromosome doubling of mitotically active 'Femminello'colonies. These autoploid lemon plants are less vigorous than diploid 'Femminello' plants and the alloploid 'Valencia' + 'Femminello' somatic hybrid plant. Because of the increased number of alleles present in an allotetraploid somatic hybrid, there is greater potential for heterosis, and this may explain the observed vigor in the interspecific 'Valencia' + 'Femminello' somatic hybrid plant.

Previously, a high percentage of seedless triploid progeny that produced high-acid fruit was obtained from a cross of tetraploid 'Lisbon' lemon with diploid 'Trovita' sweet orange (Soost and Cameron, 1969). More recently, diploid x tetraploid crosses have produced seedless triploid scion cultivars in Citrus (Soost and Cameron, 1980, 1985), but this method has been limited by the few suitable tetraploid breeding parents (Grosser and Gmitter, 1990b).

\section{Literature Cited}

Cardy, B. J., C.W. Stuber, and M.M. Goodman. 1981. Techniques for starch gel electrophoresis of enzymes from maize (Zea maize L.). Institute of Statistics, Mimeograph Series no. 1317. North Carolina State Univ., Raleigh.

Grosser, J.W. and J.L. Chandler. 1987. Aseptic isolation of leaf protoplasts from Citrus, Poncirus, Citrus x Poncirus hybrids and Severinia for use in somatic hybridization experiments. Scientia Hort. $31: 253-257$.

Grosser, J.W. and F.G. Gmitter, Jr. 1990a. Somatic hybridization of Citrus with wild relatives for germplasm enhancement and cultivar development. HortScience 25:147-151.

Grosser, J.W. and F.G. Gmitter, Jr. 1990b. Protoplasm fusion and citrus improvement. Plant Breed Rev. 8:339-374.

Grosser, J. W., F.G. Gmitter, Jr., and J.L. Chandler. 1988a. Intergeneric somatic hybrid plants of Citrus sinensis cv. Hamlin and Poncirus trifoliata cv. Flying Dragon. Plant Cell Rpt. 7:5-8.

Grosser, J. W., F.G. Gmitter; Jr., and J.L. Chandler. 1988b. Intergeneric somatic hybrid plants from sexually incompatible woody species: Citrus sinensis and Severinia disticha. Theor. Applied Genet. 75:397-401.

Grosser, J. W., G. A. Moore, and F.G. Gmitter, Jr. 1989. Interspecific somatic hybrid plants from the fusion of 'Key' lime (Citrus aurantifolia) with 'Valencia' sweet orange (Citrus sinensis) protoplasts. Scientia Hort. 39:23-29.

Kobayashi, S. and T. Ohgawara. 1988. Production of somatic hybrid plants through protoplasm fusion in citrus. J. Agr. Rev. Quart. 22:181188 .
Kobayashi, S., T. Ohgawara, E. Ohgawara, I. Oiyama, and S. Ishii. 1988. A somatic hybrid plant obtained by protoplasm fusion between navel orange (Citrus sinensis) and satsuma mandarin. Plant Cell Tissue Organ Culture 14:63-69.

Menczel, L., F. Nagy, Z. Kiss, and P. Maliga. 1981. Streptomycin resistant and sensitive hybrids of Nicotiana tabacum and Nicotiana knightiana: Correlation of resistance with N. tabacum plastids. Theor. Applied Genet. 59:191-195.

Moore, G.A. and W.S. Castle. 1988. Morphological and isozymic analysis of open-pollinated Citrus rootstock populations. J. Hered. 79:59-63.

Murashige, T. and D.P.H. Tucker. 1969. Growth factor requirements of citrus tissue culture. Proc. Ist Intl. Citrus Symp. 3: 1155-1 161.

Ohgawara, T., S. Kobayashi, S. Ishii, K. Yoshinaga, and I. Oiyama. 1989. Somatic hybridization in Citrus: navel orange (C. sinensis Osb.) and grapefruit (C. paradisi Macf.). Theor. Applied Genet. 78:609-612.

Ohgawara, T., S. Kobayashi, E. Ohgawara, H. Uchimiya, and S. Ishii. 1985. Somatic hybrid plants obtained by protoplasm fusion between Citrus sinensis and Poncirus trifoliata. Theor. Applied Genet. 71:14.

Power, J. B., J.V. Chapman, and D. Wilson. 1984. Laboratory manual: Plant tissue culture. Plant Genetic Manipulation Group, Dept. of Botany, Univ. of Nottingham. p. 125.

Salerno, M. and G. Cutuli. 1977. Control of citrus mal secco in Italy today. Proc. Intl. Soc. Citriculture 3:1001-1003.

Sass, J.E. 1958. Botanical microtechnique. Iowa State Univ. Press, Ames. p. 73-75.

Solel, Z. and M. Salerno. 1988. Mal secco, p. 18-20. In: J.O. Whiteside, S.M. Garnsey, and L.W. Timmer (eds.). Compendium of citrus diseases. APS Press, St. Paul, Minn.

Soost, R.K. and J.W. Cameron. 1969. Tree and fruit characters of Citrus triploids from tetraploid by diploid crosses. Hilgardia 39:569577.

Soost, R.K. and J.W. Cameron. 1980. 'Oroblanco,' a triploid pummelo-grapefruit hybrid. HortScience 15:667-669.

Soost, R.K. and J.W. Cameron. 1985. 'Melogold,' a triploid pummelo-grapefruit hybrid. HortScience 20:1134-1135.

Swingle, W.T. and P.C. Reece. 1967. The botany of citrus and its wild relatives, p. 190430. In: W. Reuther, L.D. Batchelor, and H.J. Weber (eds.). The citrus industry. vol. 1. Univ. of California Press, Berkeley.

Torres, A. J., R.K. Soost, and U. Diedenhofen. 1978. Leaf isozymes as genetic markers in citrus. Amer. J. Bet. 65:869-881.

Torres, A. J., T. Mau-Lastovicka, T.E. Williams, and R.K. Soost. 1985. Segregation distortion and linkage of Citrus and Poncirus isozyme genes. J. Hered. 76:289-294.

Vallejos, C. 1983. Enzyme activity staining, p. 469-516. In: S.D. Tanksley and T.J. Orton (eds.). Isozymes in plant genetics and breeding, Part A. Elsevier, Amsterdam.

Vardi, A., P. Spiegel-Roy, and E. Galun. 1982. Plant regeneration from citrus protoplasts: variability in methodological requirements among cultivars and species. Theor. Applied Genet. 62:171-176. 